\title{
ARTICLE OPEN \\ Scalable ion-photon quantum interface based on integrated diffractive mirrors
}

Moji Ghadimi ${ }^{1}$, Valdis Blūms ${ }^{1}$, Benjamin G. Norton ${ }^{1}$, Paul M. Fisher ${ }^{1}$, Steven C. Connell ${ }^{1}$, Jason M. Amini ${ }^{2}$, Curtis Volin ${ }^{2}$, Harley Hayden ${ }^{2}$, Chien-Shing Pai ${ }^{2}$, David Kielpinski ${ }^{1}$, Mirko Lobino (iD ${ }^{1,3}$ and Erik W. Streed (iD ${ }^{1,4}$

Quantum networking links quantum processors through remote entanglement for distributed quantum information processing and secure long-range communication. Trapped ions are a leading quantum information processing platform, having demonstrated universal small-scale processors and roadmaps for large-scale implementation. Overall rates of ion-photon entanglement generation, essential for remote trapped ion entanglement, are limited by coupling efficiency into single mode fibers and scaling to many ions. Here, we show a microfabricated trap with integrated diffractive mirrors that couples $4.1(6) \%$ of the fluorescence from a ${ }^{174} \mathrm{Yb}^{+}$ion into a single mode fiber, nearly triple the demonstrated bulk optics efficiency. The integrated optic collects $5.8(8) \%$ of the $\pi$ transition fluorescence, images the ion with sub-wavelength resolution, and couples $71(5) \%$ of the collected light into the fiber. Our technology is suitable for entangling multiple ions in parallel and overcomes mode quality limitations of existing integrated optical interconnects.

npj Quantum Information (2017)3:4 ; doi:10.1038/s41534-017-0006-6

\section{INTRODUCTION}

A small chain of trapped ions, confined along the node of an oscillating electric field in a Paul trap, provides a well controlled quantum system that can be cooled to the quantum ground state and precisely manipulated with lasers and microwaves. ${ }^{1-8}$ The ions are simultaneously strongly coupled to each other through the Coulomb force, and decoupled from the surrounding environment. The strong mutual coupling is critical for implementing deterministic multi-qubit entangling gates, ${ }^{9,} 10$ while the external decoupling enables memory coherence times approaching $1 \mathrm{~min}^{2}$ and single qubit gate error rates ${ }^{11}$ below $10^{-4}$. Smallscale quantum processors can be linked together into quantum networks ${ }^{1}$ using remote entanglement ${ }^{4}$ to realise the proposed roadmap for large-scale quantum information processing ${ }^{3}$. Recent developments include implementation of the Shor factoring algorithm $^{12}$ and a programmable quantum computer module based on five ions. ${ }^{13}$ In these experiments, the ions' fluorescence was collected using complex multi-element bulk optics, which may be less suitable for scaling to massively parallel systems.

Ion fluorescence plays two complementary roles in quantum information processing: state readout through the collection of multiple photons, and the creation of remote entanglement through entanglement swapping of photons coupled into single optical modes. The fidelity and readout speed for qubit state detection in trapped ion QIP depends only on the fluorescence collection efficiency. ${ }^{14}$ While efficient coupling into single-mode structures, including optical fibers or arrays of waveguides for collection from multiple ions, ${ }^{15}$ requires a collection apparatus that is also capable of producing a high quality ion image. Collection efficiencies in free space of up to $54.8 \%{ }^{16}$ have been realised in custom fabricated parabolic mirror with very large solid angle coverage. However these devices are highly labor intensive to construct and not scalable. A more scalable approach used reflected curved surface optics into microfabricated ion traps, ${ }^{8}$ but the ion image quality of such system remains insufficient for good single mode coupling. In fact for both local processing and remote networking, a scalable optical interface that can efficiently interface multiple ions with single mode guiding structures is necessary to achieve large, massively parallel implementations.

Diffractive optics offers a solution to this problem since they are scalable, can engineer out geometrical aberrations at the design phase for efficient coupling into single mode structures, and are compatible with several platform such as neutral atom chip traps ${ }^{17}$ or crystal color centers. ${ }^{18}$ Previous efforts using diffractive lenses with traditional macro traps have demonstrated a $4.2 \%$ collection efficiency ${ }^{19}$ in free space as well as near diffractionlimited imaging in both fluorescence ${ }^{20}$ and absorption ${ }^{21}$ modalities, the latter being important for implementing quantum photonic receivers without the need for ion-photon interaction enhancement by an optical cavity. ${ }^{22}$

\section{RESULTS}

Here, we exploit the potential of diffractive optics and demonstrate a scalable photon-ion interface realised on a multi-zone micro-fabricated surface trap with integrated diffractive mirrors. Our trap is based on a proven design ${ }^{23}$ and is shown in Fig. 1 a. The substrate under the central grounding electrode was patterned and etched before coating with $100 \mathrm{~nm}$ of aluminum to also act as an array of diffractive mirrors (see Methods). We tested the properties of the integrated mirror by measuring its collection efficiency with a protocol for the creation of triggered single photons and acquiring a near diffraction-limited image of a single ion. With such image quality we were able to obtained an

\footnotetext{
${ }^{1}$ Centre for Quantum Dynamics, Griffith University, Brisbane, QLD 4111, Australia; ${ }^{2}$ Georgia Tech Research Institute, Atlanta, GA 30318 , USA; ${ }^{3}$ Queensland Micro and Nanotechnology Center, Griffith University, Brisbane, QLD 4111, Australia and ${ }^{4}$ Institute for Glycomics, Griffith University, Gold Coast, QLD 4222, Australia Correspondence: Erik W. Streed (e.streed@griffith.edu.au)
}

Received: 12 August 2016 Revised: 1 December 2016 Accepted: 15 December 2016 Published online: 31 January 2017 
overall coupling efficiency from ion into single mode fiber which doubled what was previously achieved with micro-trap and multimode fibers by direct collection ${ }^{6}$ and with the use of diffractive lenses ${ }^{7}$ respectively.

The diffractive mirror has a focal length of $59.6 \mu \mathrm{m}$ which correspond to the ion height necessary for high resolution
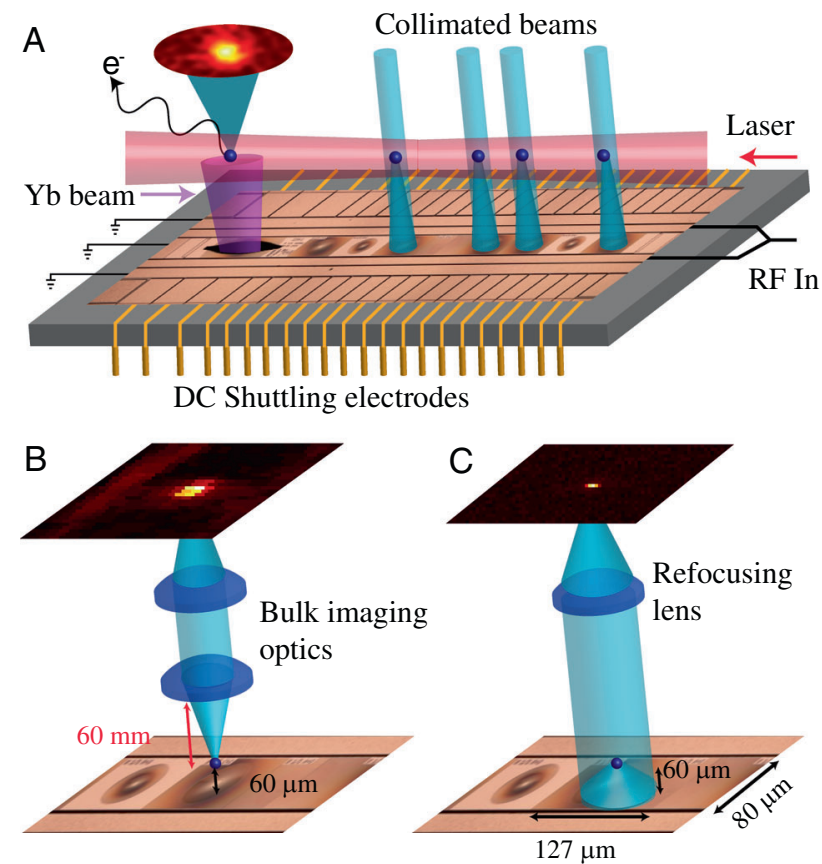

Fig. 1 a Schematic of the surface ion trap modified with an array of integrated monolithic diffractive mirrors. Neutral $\mathrm{Yb}$ from a thermal beam (purple, left) is photo-ionized in a loading zone, laser cooled, and the ${ }^{174} \mathrm{Yb}^{+}$ion (blue dots) is shuttled to one of the collimator sites by modulating the DC electrode array. Illustrated laser beam (pink) orientation is for clarity, actual is coplanar to chip surface and $45^{\circ}$ to the RF rails. b Conventional ion fluorescence imaging using external bulk optics with $1 \%$ collection efficiency. c Diffractive mirror imaging with $5.8(8) \%$ collection efficiency of the $\pi$ transition fluorescence imaging, when the collimated flourescence is refocused by an external lens at the desired magnification (Fig. 1c). The integrated optic has a design diffraction efficiency of $50 \%$, which includes $92 \%$ aluminum reflectivity in the ultra-violet, and is $80 \mu \mathrm{m}$ wide by $127 \mu \mathrm{m}$ long. The width of the mirror is limited by the center ground electrode size and the length is set to match the industry standard $127 \mu \mathrm{m}$ pitch of V-groove fiber arrays for simultaneous coupling of multiple ions. The diffractive mirror has a numerical aperture (NA) of 0.55 in the $80 \mu \mathrm{m}$ direction and 0.73 in the 127 $\mu \mathrm{m}$ direction parallel to the radiofrequency (RF) rails, capturing $13.3 \%$ of the total solid angle. This is equivalent to a circular NA of 0.68 , which maximizes the overall rate of entanglement generation per unit surface area of the trap. ${ }^{15}$

The trap is loaded with ${ }^{174} \mathrm{Yb}^{+}$ions by isotope selective photoionization from an effusive $\mathrm{Yb}$ beam passing through a slot in the chip surface (Fig. 1a farthest left ion image). Trapped ions are Doppler cooled and shuttled from the loading zone to the various diffractive optic focus sites by varying the potential in the segmented DC voltage electrode arrays. A conventional bulk optics imaging system (Fig. 1b) allows the ion to be observed at all points along the RF rail for diagnostic purposes. As an indication of the robustness of diffractive optics as an imaging solution, a test diffractive mirror patterned around the $\mathrm{Yb}$ oven loading slot was successfully used to image ions, despite the large central void which reduced its collection efficiency. A magnetic field coplanar with the chip, at $45^{\circ}$ to the RF rails, sets the direction of the quantization axis. The laser's direction is aligned with the magnetic field such that circularly polarized light excites either the $\sigma^{+}$or $\sigma^{-}$transitions.

We measured the collection efficiency of the integrated optics using a single photon generation protocol based on opticalpumping (see Fig. 2 and Methods). The protocol relies on selective illumination with $\sigma^{+}$and $\sigma^{-}$polarized lasers followed by emission of a $\pi$ photon which pumps the atom into a dark ground state (Fig. 2a, b). Differences in the radiation distribution patterns results in our optic collecting $17.4 \%$ of the fluorescence from $\pi$ polarized and $11.3 \%$ from $\sigma$ polarized transitions. In the direction perpendicular to the quantization axis and parallel to the collection optic axis, $\pi$ and $\sigma$ photons are orthogonally polarized. With this geometry, single $\pi$ photons were selectively detected placing a linear polariser before a photomultiplier tube (PMT). To verify the integrity of our single photon generation protocol, we
A

Cooling Preparation

\begin{tabular}{|c|c|c|c|c|c|c|c|}
\hline Time(ns) & 500 & 500 & 750 & 250 & 250 & 500 & 500 \\
\hline$\sigma=$ & & & & & & & \\
\hline$\sigma+$ & & & & & & & \\
\hline Detection & & & & & & & \\
\hline
\end{tabular}

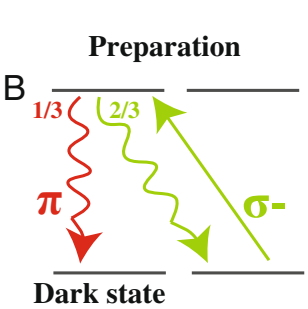

Single photon

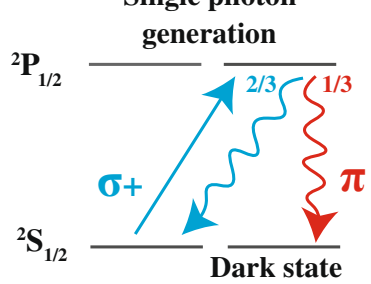

C
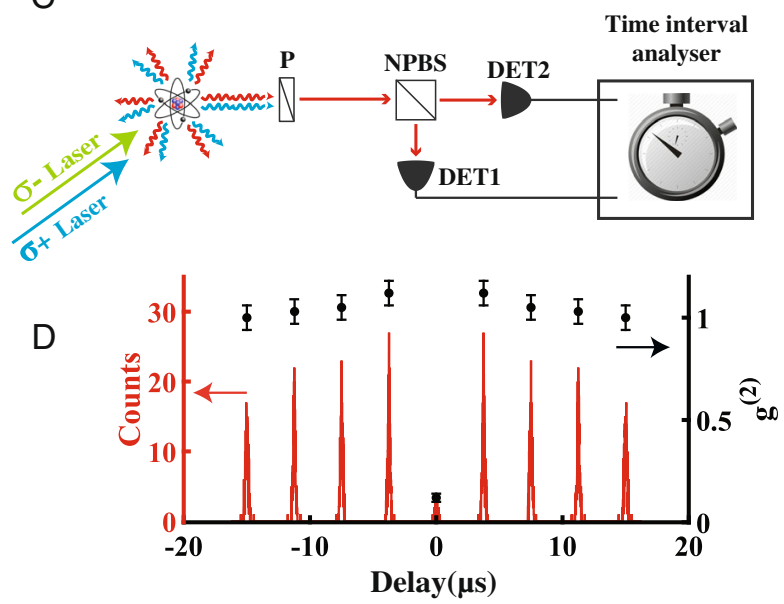

Fig. 2 Single photon generation protocol. a Pulse sequence for $\sigma^{+}$and $\sigma^{-}$lasers and detection gating. b ${ }^{174} \mathrm{Yb}^{+}$level diagram showing $\pi$ photons result in optical pumping to a dark state. c Experimental set-up of the second order correlation measurement $g^{(2)}$. Ion fluorescence is analyzed with a linear polariser (P) to filter out $\sigma$ photons, leaving only $\pi$ photons to be divided by a $50 / 50$ non-polarizing beamsplitter (NPBS) between two photomultiplier tube detectors (DET1, DET2). Arrival time statistics are accumulated by a digital interval analyzer. $\mathbf{d}$ Measured coincidence counts and second-order correlation $g^{(2)}$. Peak spacing corresponds to $3.25 \mu$ s experimental repetition period 


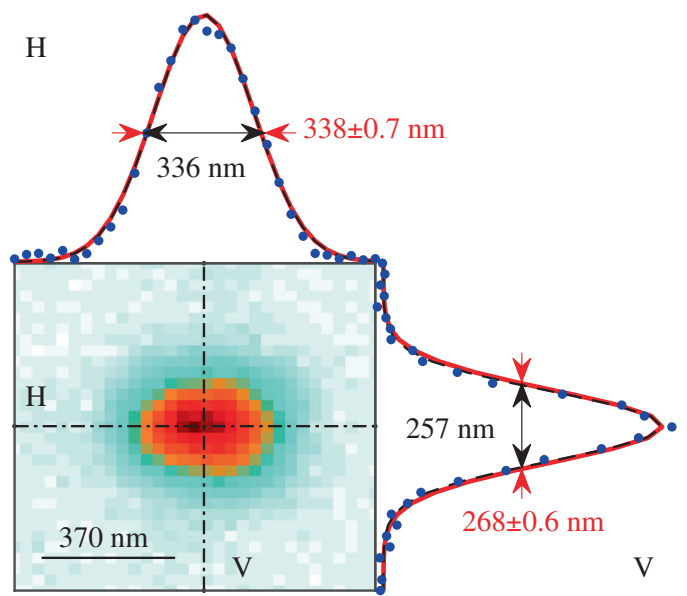

Fig. 3 Sub-wavelength image of an ${ }^{174} \mathrm{Yb}^{+}$ion by an integrated diffractive mirror. Near diffraction limited performance was obtained from a rectangular optic $80 \times 127 \mu \mathrm{m}$ operating at $\mathrm{NA}=0.56 \times 0.73$ covering a solid angle $\Omega / 4 \pi=13.3 \%$. Imaged at magnification $390 \times$, $1 \mathrm{~s}$ exposure. Horizontal $(\mathrm{H})$ and Vertical $(\mathrm{V})$ cross sections indicate image data (blue points), $338(0.7) \times 268(0.6) \mathrm{nm}$ FWHM diameter gaussian fits (red), and predicted diffraction limit (black dashed)

measured the second order correlation $g^{(2)}(\tau)$ of the fluorescence collected through the integrated mirror with a 50/50 nonpolarizing beam splitter and two detectors (Fig. 2c). Our large NA results in polarization blurring ${ }^{24}$ of the $\sigma$ photons far from the collection optic axis. To reduce the transmission of $\sigma$ photons through the polariser, we used an iris to temporarily decrease the NA from 0.68 to 0.48 and measured a $g^{(2)}(0)$ of $0.12(2)$ (Fig. $3 d$ ). This value is well below threshold of 0.5 for a single photon emitter, ${ }^{25}$ but larger than our expected residual value for $g^{(2)}(0)$ of 0.069 . The difference is likely due to residual polarization blurring, imperfections of the polarization purity of the excitation laser and background scattering of the incident laser light off the chip's surface. Residual polarization blurring is an intrinsic aspect of the dipole radiation patterns ${ }^{26}$ and protocols, which leverage time-bin or frequency encoding may be necessary to realize the full advantage of large NA optics for efficient generation of remote entanglement. The $308 \mathrm{kHz}$ repetition rate of this protocol is comparable with the present state of the art for remote entanglement ion trapping experiments. ${ }^{5}$

In order to measure the collection efficiency of the diffractive mirror, we ran the triggered single photon protocol 184,000 times and counted 770 photons. Adjusting for known loss processes (i.e. $19 \%$ detector quantum efficiency, 50(5)\% transmission through the iris, and $24 \%$ losses through other optical elements including the polariser) we measured a 5.8(8)\% collection efficiency for our diffractive mirror on the $\pi$ transition. In comparison, our theoretical efficiency of $8.7 \%$ is the product of the $17.4 \%$ solid angle coverage for the $\pi$ transition and the $50 \%$ expected diffraction efficiency. This value indicates that our diffraction efficiency is $33(7) \%$ and not the expected $50 \%$, most likely due to fabrication imperfections in the optics.

Figure 3 shows a near diffraction-limited image of an ion with a sub-wavelength spot size of 338(0.7) $\mathrm{nm}$ horizontal $(\mathrm{H})$ and 268 (0.6) $\mathrm{nm}$ vertical (V) FWHM diameter. This is only slightly larger $(0.6 \%$ for horizontal and $4 \%$ for vertical) than the diffraction limited size of $336 \mathrm{~nm}(\mathrm{H})$ and $257 \mathrm{~nm}(\mathrm{~V})$, and approaching the state of the art $280 \mathrm{~nm}$ circular spot size reported for a speciality $81 \%$ solid angle coverage parabolic reflector optic. ${ }^{16}$ The $M^{2}$ mode quality of the spot was $1.36(\mathrm{H})$ and $1.54(\mathrm{~V})$, compared to an ideal Gaussian $\left(M^{2}=1\right)$. This non-ideal behavior is driven by increased divergence in the beam due to astigmatism from the aperture and the fundamentally non-Gaussian distribution of the ion's emission.

We benchmarked the collection and imaging capabilities of the integrated mirror by coupling the ion's fluorescence into a single mode fiber. We remove the iris and polariser from the set-up and used a mode matching telescope for adjusting the average radius of the ion fluorescence image to that of a single fiber mode with an estimated spatial mode overlap of $98 \%$. The ion image's average $M^{2}$ of 1.45 reduces the predicted coupling efficiency to $68 \%$. We measured a transmission from the ion through a single mode fiber of $57(4) \%$, which combined with a $80 \%$ fiber transmission due to propagation and Fresnel losses correspond to a single mode coupling efficiency of $71(5) \%$. This value is in good agreement with the estimated $68 \%$ coupling efficiency and combined with the 5.8(8)\% $\pi$ transition collection efficiency from the diffractive optics and $8.3 \%$ losses through other optical elements, gives us a total measured coupling efficiency from ion to fiber of $4.1(6) \%$. This ion-fiber coupling efficiency is nearly triple the previous best of $1.4 \%$ using a conventional lens, ${ }^{5}$ corresponding to a 8.6 times gain in entanglement generation rate. This efficiency could be substantially improved with larger NA optics or modifying the optic's design to mode-match a specific transition's intensity distribution to a single mode fiber. More sophisticated multi-level diffraction gratings ${ }^{27}$ could improve the diffraction efficiency towards $99 \%$.

\section{DISCUSSION}

We have realised a microfabricated architecture for interfacing a single ion with single mode optical fiber and free space based on integrated diffractive mirrors, which is in-principle scalable to a large number of sites. Using a triggered single photon generation protocol, we measured a collection efficiency for the integrated optics of $5.8(8) \%$ a coupling efficiency into a single mode optical fiber of $71(5) \%$. These mirrors are monolithic with the metallic trap electrode, cover a large solid angle, and can be within a few tens of microns from the ion without distorting the RF trapping potential. They are therefore an ideal platform for the implementation of quantum networks and remote entanglement sharing with trapped ions but also with other quantum light sources such as neutral atom chip traps ${ }^{17}$ or fixed emitters such as crystal color centers like $\mathrm{NV}^{-}$in diamond. ${ }^{18}$

\section{METHODS}

\section{Trap fabrication}

The micro-fabrication procedure ${ }^{23}$ for the surface trap includes depositing multiple layers of aluminum $(R=92 \%, \lambda=370 \mathrm{~nm})$ separated by silicon dioxide insulating layers of various thicknesses. In order to incorporate the diffractive optical elements into this procedure, the oxide surface separating the control electrodes from the ground layer was patterned using e-beam lithography and reactive ion etching of the oxide. The patterned area was subsequently metallized with a $100 \mathrm{~nm}$ thick layer of aluminum and the chip was inspected to ensure that the contours of the diffractive optic had not compromised the electrical integrity of the ground electrode. The groove step design is a hybrid of a four level grating near the center with low spatial period and a two level grating near the edges where there is a high spatial period. Grating height step size is 45 $\mathrm{nm}$ for 4 level area and $90 \mathrm{~nm}$ for 2 level area. Approximating small chunks of the grating as 1D structures, the blazing profile was optimized in GSolver to account for the finite height of the grating and vector diffraction effects.

Fabrication imperfections can cause a mismatch between the optic focal point and the expected RF node altitude of $58.6 \mu \mathrm{m}$ above the surface of the trap. This is a critical matching problem due to the small depth of focus in low aberration, large aperture ion imaging. ${ }^{20}$ To guard against this possibility our five collimating optics were fabricated with focal lengths ranging from $f=58.6$ to $62.6 \mu \mathrm{m}$ in $1 \mu \mathrm{m}$ steps. Stray electrical fields from the neighboring oven loading zone precluded the use of the nominal $f_{0}=$ $58.6 \mu \mathrm{m}$ mirror site and instead experiments were performed on the $f_{+1 \mu \mathrm{m}}$ 
$=59.6 \mu \mathrm{m}$ focal length collimator. A DC potential was applied to shift the ion off the RF node and position it at the focal point. We observed it was more reliable to pull the ion towards the surface of the trap using a DC potential, rather than pushing it away the trap. While we did not observe a mismatch, for higher NA this parameter will become even more stringent. In future iterations this could be corrected dynamically with additional RF lines or statically by laser trimming the RF electrodes.

\section{Triggered single photon generation protocol}

To measure the collection efficiency of the integrated mirror we implemented an optical pumping based single photon generation protocol (Fig. 2). The protocol relies on decay into a dark optically pumped state requiring the emission of a terminal $\pi$ polarized photon rather than a $\sigma$ polarized photon (Fig. 2b). The ion was first Doppler cooled for $500 \mathrm{~ns}$ with two laser beams of $\sigma^{+}$and $\sigma^{-}$polarizations with $7 \mu \mathrm{W}$ and $100 \mu \mathrm{m}$ diameter at $370 \mathrm{~nm}$, detuned $-10 \mathrm{MHz}$ from resonance. Each scattered photon has a $2 / 3$ chance of returning to its original ground state via a $\sigma$ decay, or a $1 / 3$ chance of being optically pumped into the other ground state via a $\pi$ transition. The ion was then optically pumped in the ${ }^{2} S_{1 / 2} m_{\mathrm{F}}=+1 / 2$ state by $500 \mathrm{~ns}$ illumination with just the $\sigma^{-}$light. $750 \mathrm{~ns}$ of wait time was introduced for acousto-optic modulator switching time to ensure all laser beams were off. The detection window was then activated for $1000 \mathrm{~ns}$ with a $250 \mathrm{~ns}$ pulse of $\sigma^{+}$occurring $250 \mathrm{~ns}$ into the detection window. On average this process takes three scattering events $(2 \sigma$ and $1 \pi)$ and the whole duration of one cycle is $3.25 \mu \mathrm{s}$. Since the collection optic was oriented perpendicular to the quantization axis, the $\sigma$ and $\pi$ photons had perpendicular linear polarizations allowing us to filter out the single $\pi$ photon with a linear polariser and detect it on the PMT. Occasional decays $(0.5 \%)$ into the ${ }^{2} D_{[3 / 2]}$ dark state were repumped out with $650 \mu \mathrm{W}$ of 935 $\mathrm{nm}$ light and did not have a meaningful impact on the experiment.

\section{ACKNOWLEDGEMENTS}

The phase Fresnel mirrors were fabricated by M. Ferstl at the Heinrich-Hertz-Institut of the Fraunhofer-Institut für Nachrichtentechnik in Germany. This material is based upon work supported by the Office of the Director of National Intelligence (ODNI), Intelligence Advanced Research Projects Activity (IARPA) under U.S. Army Research Office (ARO) contract number W911NF1210600. All statements of fact, opinion, or conclusions contained herein are those of the authors and should not be construed as representing the official views or policies of IARPA, the ODNI, or the U.S. Government. This work was supported by the Australian Research Council (ARC) under DP130101613. D.K. was supported by ARC Future Fellowship FT110100513. E. W.S was supported by ARC Future Fellowship FT130100472. M.L. was supported by ARC-DECRA DE130100304.

\section{AUTHOR'S CONTRIBUTIONS}

Ion trap construction and experimental work was performed by M. Ghadimi, V. Blūms, B. G. Norton, P. M. Fisher, S. C. Connell, and D. Kielpinski with supervision by D. Kielpinski, M. Lobino, and E.W. Streed. The chip trap was fabricated by J. M. Amini, C. Volin, H. Hayden, and C. S. Pai at Georgia Tech Research Institute. The manuscript was prepared by M. Ghadimi, M. Lobino, and E.W. Streed in consultation with the other authors.

\section{COMPETING INTERESTS}

The authors declare no competing interests.

\section{REFERENCES}

1. Kimble, H. J. The quantum internet. Nature 453, 1023-1030 (2008).

2. Harty, T. P. et al. High-fidelity preparation, gates, memory, and readout of a trapped-ion quantum bit. Phys. Rev. Lett. 113, 220501 (2014).

3. Monroe, C. et al. Large-scale modular quantum-computer architecture with atomic memory and photonic interconnects. Phys. Rev. A 89, 022317 (2014).

4. Bouwmeester, D. et al. Experimental quantum teleportation. Nature 390, 575-579 (1997).
5. Hucul, D. et al. Modular entanglement of atomic qubits using photons and phonons. Nat. Phys. 11, 37-42 (2015).

6. VanDevender, A. P., Colombe, Y., Amini, J., Leibfried, D. \& Wineland, D. J. Efficient fiber optic detection of trapped ion fluorescence. Phys. Rev. Lett. 105, 023001 (2010).

7. Clark, C. R. et al. Characterization of fluorescence collection optics integrated with a microfabricated surface electrode ion trap. Phys. Rev. Appl. 1, 024004 (2014).

8. Merrill, J. T. et al. Demonstration of integrated microscale optics in surfaceelectrode ion traps. New J. Phys. 13, 103005 (2011).

9. Cirac, J. \& Zoller, P. Quantum computations with cold trapped ions. Phys. Rev. Lett. 74, 4091 (1995).

10. Mølmer, K. \& Sørensen, A. Multiparticle entanglement of hot trapped ions. Phys. Rev. Lett. 82, 1835-1838 (1999).

11. Brown, K. R. et al. Single-qubit-gate error below $1 \mathbf{1 0}^{-\mathbf{4}}$ in a trapped ion. Phys. Rev. A 84, 030303 (2011).

12. Monz, T., Chuang, I. L. \& Blatt, R. Realization of a scalable shor algorithm. Science 351, 1068 (2016).

13. Debnath, S. et al. Demonstration of a programmable quantum computer module. Nature 536, 63-66 (2016).

14. Wölk, S., Piltz, C., Sriarunothai, T. \& Wunderlich, C. State selective detection of hyperfine qubits. J. Phys. B 48, 075101 (2015).

15. Kielpinski, D., Volin, C., Streed, E. W., Lenzini, F. \& Lobino, M. Integrated optics architecture for trapped-ion quantum information processing. Quantum Inf. Process 1-24 (2015). http://link.springer.com/article/10.1007/s11128-0151162-2.

16. Maiwald, R. et al. Collecting more than half the fluorescence photons from a single ion. Phys. Rev. A 86, 043431 (2012). http://link.aps.org/doi/10.1103/ PhysRevA.86.043431.

17. Folman, R. et al. Controlling cold atoms using nanofabricated surfaces: atom chips. Phys. Rev. Lett. 84, 4749-4752 (2000). http://link.aps.org/doi/10.1103/ PhysRevLett.84.4749.

18. Marseglia, L. et al. Nanofabricated solid immersion lenses registered to single emitters in diamond. Appl. Phys. Lett. 98 , 133107 (2011). http://aip.scitation.org/ doi/10.1063/1.3573870.

19. Streed, E. W. et al. Imaging of trapped ions with a microfabricated optic for quantum information processing. Phys. Rev. Lett. 106, 010502 (2011).

20. Jechow, A., Streed, E. W., Norton, B. G., Petrasiunas, M. J. \& Kielpinski, D. Wavelength-scale imaging of trapped ions using a phase fresnel lens. Opt. Lett. 36, 1371-1373 (2011).

21. Streed, E. W., Jechow, A., Norton, B. G. \& Kielpinski, D. Absorption imaging of a single atoms. Nat. Commun. 3, 933 (2012).

22. Lamata, L. et al. Ion crystal transducer for strong coupling between single ions and single photons. Phys. Rev. Lett. 107, 030501 (2011). http://link.aps.org/doi/ 10.1103/PhysRevLett.107.030501.

23. Shappert, C. M. et al. Spatially uniform single-qubit gate operations with nearfield microwaves and composite pulse compensation. New J. Phys. 15, 083053 (2013).

24. Blinov, B. B., Moehring, D. L., Duan, L.-M. \& Monroe, C. Observation of entanglement between a single trapped atom and a single photon. Nature 428, 153-157 (2004).

25. Loudon, R. The Quantum Theory of Light, 3rd edn (Oxford University Press, 2000).

26. Streed, E. W., Norton, B. G., Chapman, J. J. \& Kielpinski, D. Scalable, efficient ion-photon coupling with phase fresnel lenses for large-scale quantum computing. Quantum Inf. Comput 9, 0203-0214 (2009).

27. Destouches, N., Tishchenko, A. V., Pommier, J. C., Reynaud, S. \& Parriaux, O. $99 \%$ efficiency measured in the 1 st order of a resonant grating. Opt. Express 13, 3230 (2005).

This work is licensed under a Creative Commons Attribution 4.0 International License. The images or other third party material in this article are included in the article's Creative Commons license, unless indicated otherwise in the credit line; if the material is not included under the Creative Commons license, users will need to obtain permission from the license holder to reproduce the material. To view a copy of this license, visit http://creativecommons.org/licenses/by/ $4.0 /$

(c) The Author(s) 2017 